\section{ORIGINAL RESEARCH}

K. Šurlan-Popovič
S. Bisdas
Z. Rumboldt
T.S. Koh
P. Strojan

\title{
Changes in Perfusion CT of Advanced Squamous Cell Carcinoma of the Head and Neck Treated during the Course of Concomitant Chemoradiotherapy
}

BACKGROUND AND PURPOSE: Concomitant chemoradiation is a promising therapy for the treatment of locoregionally advanced head and neck carcinoma. The purpose of this study was to prospectively evaluate early changes in primary tumor perfusion parameters during concomitant cisplatin-based chemoradiotherapy of locoregionally advanced SCCHN and to evaluate their predictive value for response of the primary tumor to therapy.

MATERIALS AND METHODS: Twenty patients with locoregionally advanced SCCHN underwent perfusion CT scans before therapy and after completion of $40 \mathrm{~Gy}$ and $70 \mathrm{~Gy}$ of chemoradiotherapy. BF, BV, MTT, and PS of primary tumors were quantified. Differences in perfusion and tumor volume values during the therapy as well as between responders and nonresponders were analyzed, and ROC curves were used to assess predictive value of the baseline and follow-up functional parameters.

RESULTS: The tumor volumes at $40 \mathrm{~Gy}$ and at $70 \mathrm{~Gy}$ were significantly lower compared with baseline values $(P=.014$ and $P=.007)$. In the 6 nonresponders, measurements after 40 Gy showed a nonsignificant trend of increased BF, BV, and PS values compared with the baseline values $(P=.06)$. In 14 responders, a significant reduction of $\mathrm{BF}$ values was recorded after $40 \mathrm{~Gy}(P=.04)$ and after $70 \mathrm{~Gy}(P=$ .01). In responders, BV values showed a reduction after $40 \mathrm{~Gy}$ followed by a plateau after $70 \mathrm{~Gy}(P=.04)$, whereas in nonresponders there was a nonsignificant elevation of the BV. Baseline BV predicted shortterm tumor response with a sensitivity of $60 \%$ and specificity of $100 \%(P=.01)$. After completion of 40 Gy of concomitant chemoradiation BV was a more significant predictor than were BF and MTT.

CoNCLUSIONS: The results suggest that in advanced SCCHN the perfusion CT monitoring might be of predictive value for identifying tumors that may respond to cisplatin-based chemoradiotherapy.

\begin{abstract}
ABBREVIATIONS: $\mathrm{AUC}=$ area under the curve; $\mathrm{BF}=$ blood flow; $\mathrm{BV}=$ blood volume; $\mathrm{CECT}=$ contrast-enhanced $\mathrm{CT}$; $\mathrm{LR}=$ likelihood ratio; $\mathrm{MTT}=$ mean transit time; $\mathrm{PCT}=$ perfusion $\mathrm{CT}$; $\mathrm{PET}=$ positron-emission tomography; PS = permeability surface area product; $\mathrm{ROC}=$ receiver operating characteristic; $\mathrm{ROI}$ = region of interest; $\mathrm{SCCHN}=$ squamous cell carcinoma of the head and neck; VEGF = vascular endothelial growth factor
\end{abstract}

$\mathbf{S}^{2}$ quamous cell carcinoma of the head and neck reflects a complexity of genetic, biologic, histopathologic, and clinical heterogeneity. ${ }^{1}$ Because the primary pattern of failure in SCCHN is locoregional, surgery and radiation therapy are recognized as key treatment modalities in early tumors. In locoregionally advanced tumors, however, improved treatment outcome was observed when adding platinum-based chemotherapy concomitantly to irradiation, which translated to an absolute survival benefit of $4 \%$ at 5 years. ${ }^{2,3}$

PCT studies may delineate $\mathrm{SCCHN}^{4,5}$ and predict response to radiation therapy ${ }^{6,7}$ as well as to induction chemothera-

\section{Received May 7, 2009; accepted after revision August 6 .}

From the Department of Radiology (K.S.-P.), University Clinical Centre, Ljubljana, Slovenia; Department of Neuroradiology (Z.R.), Medical University of South Carolina, Charleston, South Carolina; Center for Modeling and Control of Complex Systems (T.S.K.), Nanyang University, Singapore; Department of Radiation Oncology (P.S.), Department of Oncology, Ljubljana, Slovenia; and Department of Neuroradiology (S.B.), Eberhard Karls University, Tübingen, Germany.

K.S.-P. and S.B. contributed equally to preparing and writing the manuscript.

Please address correspondence to Sotirios Bisdas, MD, Department of Neuroradiology, Eberhard Karls University, Hoppe-Seyler-Str 3, D-72076 Tübingen, Germany; e-mail: Sotirios.Bisdas@med.uni-tuebingen.de

DOI 10.3174/ajnr.A1859 py. ${ }^{8,9}$ However, the studies published thus far are solely based on the admission of PCT examinations (and their predictive value to specific clinical end points) and have not assessed the value of PCT as a therapeutic monitoring tool, nor they have assessed perfusion changes after completion of the therapy in patients treated exclusively with induction chemotherapy. ${ }^{6,8}$ Thus, the time course of changes in tumor parameters during concomitant chemoradiotherapy has not been investigated yet. Studying dynamics of tumor parameters might be of importance to understand the therapy-induced functional changes in the tumor tissue, to help distinguish therapy-induced alterations in responders and nonresponders, and to establish and validate PCT as a monitoring imaging technique.

In the present study we addressed these issues by prospectively examining patients with locoregionally advanced SCCHN before, during, and after the completion of concomitant chemoradiotherapy. Our primary end point was to test the hypothesis that PCT can detect changes in perfusion parameters during the course of the therapy. The secondary end points were the predictive value of the perfusion-associated parameters as measured at different time points during the therapy, as well as the correlation between the functional and morphologic CT changes. 


\begin{tabular}{lccc}
\hline \multicolumn{3}{l}{ Table 1: Demographic and clinical data } & \\
\hline Patient & $\begin{array}{c}\text { Sex/Age, } \\
\text { Years }\end{array}$ & TNM & Tumor Site \\
\hline 1 & $\mathrm{M} / 74$ & T3N2b & Oropharynx \\
2 & $\mathrm{M} / 52$ & T3N2C & Oropharynx \\
3 & $\mathrm{M} / 49$ & T4N2b & Oral cavity \\
4 & $\mathrm{M} / 69$ & T2N2c & Hypopharynx \\
5 & $\mathrm{M} / 47$ & T4N2c & Oropharynx and \\
& & & oral cavity \\
6 & $\mathrm{M} / 59$ & T4N2c & Oral cavity \\
7 & $\mathrm{M} / 52$ & T3N2b & Oropharynx \\
8 & $\mathrm{M} / 66$ & T4N2a & Oropharynx \\
9 & $\mathrm{M} / 65$ & T3N0 & Hypopharynx \\
10 & $\mathrm{M} / 56$ & T4NO & Hypopharynx \\
11 & $\mathrm{M} / 59$ & T4N3 & Oropharynx \\
12 & $\mathrm{~F} / 70$ & T4N1 & Oropharynx and \\
& & & oral cavity \\
13 & $\mathrm{M} / 60$ & T4N2c & Oropharynx and \\
& & & hypopharynx \\
14 & $\mathrm{M} / 64$ & T4N2 & Hypopharynx \\
15 & $\mathrm{M} / 62$ & T3N0 & Hypopharynx \\
16 & $\mathrm{M} / 40$ & T3N1 & Oropharynx \\
17 & $\mathrm{M} / 64$ & T2N2b & Oropharynx \\
18 & $\mathrm{M} / 70$ & T4N2c & Hypopharynx \\
19 & $\mathrm{M} / 63$ & T4N2b & Hypopharynx \\
20 & $\mathrm{M} / 54$ & T4N2C & Oral cavity \\
\hline & & &
\end{tabular}

\section{Materials and Methods}

\section{Patients and Examination Flowchart}

After approval by the local national medical ethics committee, 20 patients (19 men and 1 woman), with ages from 40 to 74 years (median age, 61 years), prospectively entered the study. They were presented for imaging of histologically proved locally and/or regionally advanced SCC of the oral cavity, oropharynx, and hypopharynx and were treated with curative intent by using concomitant chemoradiotherapy. None of the patients had systemic diseases at the time of presentation. Demographic and clinical data are summarized in Table 1.

After signing an informed consent form, each patient was examined 3 times during therapy with CECT, including PCT: 1) 0-7 days before the begining of treatment to obtain the baseline values of perfusion parameters and volume of the primary tumor; 2) at 40 Gy of concomitant chemoradiation (mean $\pm \mathrm{SD}, 36 \pm 3$ days from the start of chemoradiation); and 3) on the same day after completion of therapy, ie, at $70 \mathrm{~Gy}$ (mean $\pm \mathrm{SD}, 59 \pm 4$ days from the start of chemoradiation).

Patients with decreases in tumor volume of $30 \%$ or more from baseline as measured at the third CT examination were considered early responders; patients with tumor shrinkage of less than $30 \%$ or with increases in primary tumor volume were initially considered nonresponders. The choice of the $30 \%$ cutoff value was justified by recent evidence reporting that in serial imaging during radiation therapy of locally advanced SCCHN, the patients with local control had a median tumor volume reduction of $28 \%{ }^{10}$

\section{Concomitant Chemoradiotherapy}

A 3D conformational technique was used for radiation therapy planning. ${ }^{11}$ Patients were irradiated with a 6-MV linear accelerator photon beam and daily dose of $2 \mathrm{~Gy}$, applied in 1 fraction, 5 times per week. The total dose to the primary tumor and enlarged regional lymph nodes was $70 \mathrm{~Gy}$, with a buffer zone of $60 \mathrm{~Gy}$ around larger nodal metastases, whereas clinically uninvolved lymphatic drainage basins on the neck received $50 \mathrm{~Gy}$. The spinal cord dose was limited to $50 \mathrm{~Gy}$. During radiation therapy, cisplatin was administered in a dose of $30 \mathrm{mg} / \mathrm{m}^{2} / \mathrm{wk}$. No antiangiogenic agents were added to the treatment.

\section{CT Imaging Protocol}

PCT imaging was performed by using a 16-row multisection CT scanner (Somatom 16; Siemens, Erlangen, Germany). The scan region was based on the clinical information and the area with anatomic distortion as seen on the noncontrast "scout" 5 -mm contiguous sections $(120 \mathrm{kV}, 180 \mathrm{~mA})$. The scanned region with PCT consisted of 4 adjacent 6-mm-thick sections. For lesions larger than $24 \mathrm{~mm}$ in diameter, the levels with the largest tumor diameter were selected. The contrast agent (Iomeron 400; Bracco, Milan, Italy) for perfusion imaging (40 $\mathrm{mL}$ of $400 \mathrm{mg} / \mathrm{dL}$ nonionic iodinated contrast agent) was injected at a rate of $6 \mathrm{~mL} / \mathrm{s}$ by using a power injector and a 16-G antecubital cannula. PCT scanning $(100 \mathrm{~mA}, 80 \mathrm{kV}$, section thickness of $6 \mathrm{~mm}$, rotation time 1 second, matrix $512 \times 512 \mathrm{~mm}$ ) was initiated 6 seconds after the injection start, and 4 contiguous sections of tissue were scanned every second for 55 seconds The contrast agent administration was followed by a power injection of $20 \mathrm{~mL}$ of saline (at the same injection rate). The mean effective dose of PCT imaging was $6.4 \pm 0.3 \mathrm{mSv}$.

The dynamic study was followed immediately by a diagnostic venous phase neck study (section thickness of $3 \mathrm{~mm}[16 \times 0.75 \mathrm{~mm}$ ], $120 \mathrm{kV}, 150 \mathrm{~mA}$, rotation time 0.75 second, pitch 1.35 , matrix $512 \times$ $512 \mathrm{~mm})$. This was acquired 80 seconds after intravenous contrast injection ( $90 \mathrm{~mL}$ of the same contrast agent at a flow rate of $2 \mathrm{~mL} / \mathrm{s}$ ). The follow-up studies were acquired with the same imaging parameters and at the same tumor level by using preidentified anatomic landmarks. The contrast agent was administered exactly as described above.

\section{Image Analysis}

Image analysis was performed by 2 radiologists experienced in head and neck tumors. All PCT images were analyzed on separate workstations by using commercial deconvolution-based CT perfusion software (Perfusion 3.0; GE Healthcare, Milwaukee, Wisconsin) and the body tumor perfusion algorithm. The arterial input function for the image postprocessing was placed ipsilateral to the tumor internal carotid artery. The generated maps were BF, BV, MTT, and PS. A freehand ROI was then drawn around the primary tumor periphery in each section by using an electronic cursor. Special attention was paid not to include large feeding vessels, readily recognizable necrotic tissue (low-attenuation nonenhancing areas within tumors), and surrounding normal tissue. By viewing a cine loop of the perfusion source images of the tumor margins, the degree of any patient movement or false tumor registration during acquisition were noticed. A ROI-based evaluation in the contrast-enhanced diagnostic CT was used for gross primary tumor volume calculation (tumor volume $=$ ROI $\times$ section thickness) where readily necrotic tumor parts were excluded. The mean values for the 4 tumor functional parameters and the gross primary tumor volume for each patient were acquired after averaging the values of each section following the summation-ofareas technique. To minimize recall bias, the 2 observers analyzed all studies 3 weeks after the image acquisition.

\section{Statistical Analysis}

The continuous variables are presented as means \pm SD. After accepting normality by using the Kolmogorov-Smirnov test, a $t$ test for paired samples was used to analyze the differences in PCT and tumor 

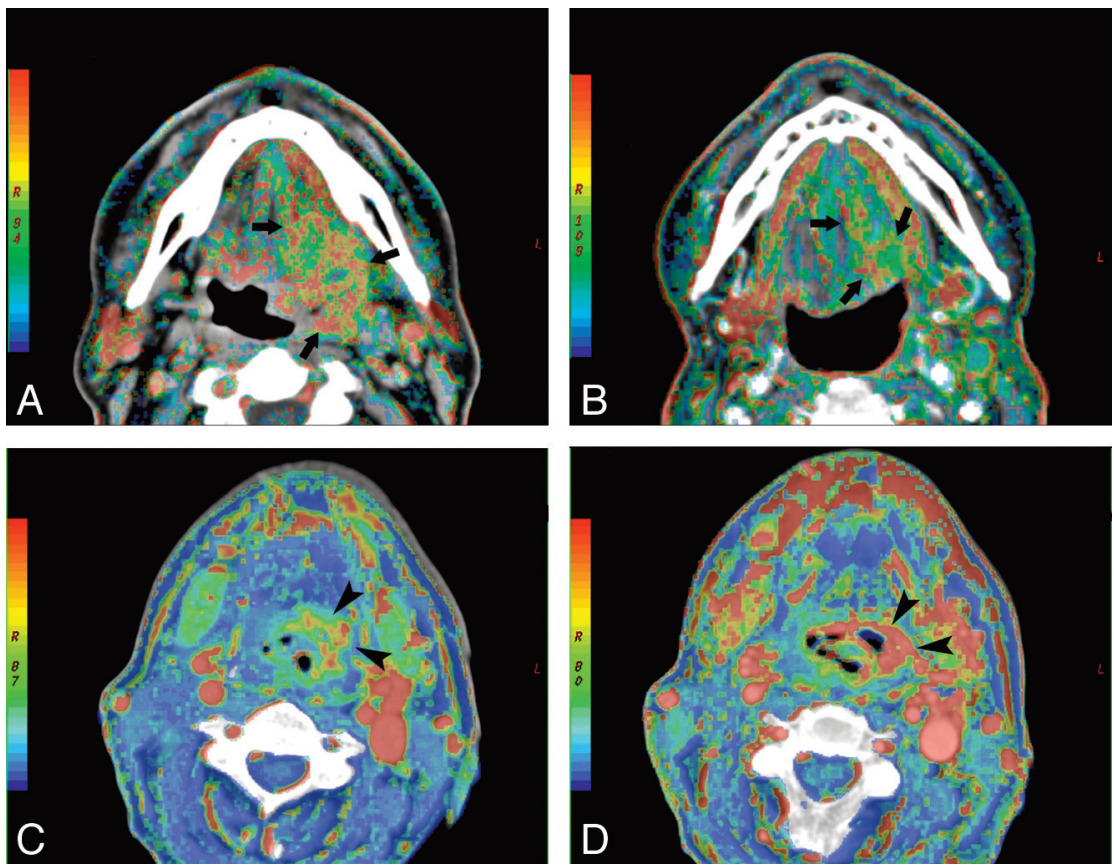

Fig 1. BF maps at admission $(A)$ and after the completion of $40 \mathrm{~Gy}(B)$ of concomitant cisplatin-based chemoradiotherapy in a responder with squamous cell carcinoma of the oropharynx (arrows). Note the significant reduction of the tumor volume at $40 \mathrm{~Gy}$, accompanied by lower BF values. Serial BV imaging at the baseline (C) and after $40 \mathrm{~Gy}(D)$ of concomitant chemoradiation in a nonresponder with squamous cell carcinoma of the hypopharynx. The BV maps demonstrate clearly the increase of BV in the tumor site during the follow-up (arrowheads)

Table 2: Perfusion parameters (mean \pm SD) in the pooled patients as well as separately in responders and nonresponders at 0,40 , and 70 Gy of concomitant chemoradiotherapy.

\begin{tabular}{|c|c|c|c|c|c|c|c|c|c|}
\hline & \multicolumn{3}{|c|}{$0 \mathrm{~Gy}$} & \multicolumn{3}{|c|}{$40 \mathrm{~Gy}$} & \multicolumn{3}{|c|}{$70 \mathrm{~Gy}$} \\
\hline & $\begin{array}{l}\text { Pooled } \\
\text { Patients }\end{array}$ & Responders & Nonresponders & $\begin{array}{l}\text { Pooled } \\
\text { Patients }\end{array}$ & Responders & Nonresponders & $\begin{array}{l}\text { Pooled } \\
\text { Patients }\end{array}$ & Responders & Nonresponders \\
\hline$\overline{\mathrm{BF}}(\mathrm{mL} / \mathrm{min} / 100 \mathrm{~g})$ & $70.4 \pm 7.9$ & $72.6 \pm 9.1$ & $69.7 \pm 7.4$ & $76.2 \pm 19.1$ & $67.5 \pm 5.9$ & $87.7 \pm 23.1$ & $66.1 \pm 28.1$ & $53.0 \pm 15.1$ & $87.8 \pm 30.1$ \\
\hline $\mathrm{BV}(\mathrm{mL} / 100 \mathrm{~mL})$ & $6.1 \pm 1.9$ & $6.4 \pm 1.4$ & $7.3 \pm 2.8$ & $6.9 \pm 2.2$ & $5.1 \pm 1.0$ & $8.5 \pm 3.2$ & $6.5 \pm 2.9$ & $5.0 \pm 1.4$ & $8.3 \pm 2.4$ \\
\hline $\operatorname{MTT}(s)$ & $8.2 \pm 3.9$ & $7.6 \pm 3.2$ & $9.3 \pm 4.1$ & $7.8 \pm 2.7$ & $6.9 \pm 2.4$ & $9.1 \pm 4.0$ & $9.9 \pm 2.7$ & $9.6 \pm 2.7$ & $10.1 \pm 4.2$ \\
\hline $\begin{array}{l}\text { Permeability } \\
\qquad(\mathrm{mL} / \mathrm{min} / 100 \mathrm{~g})\end{array}$ & $14.0 \pm 4.8$ & $14.1 \pm 3.3$ & $13.1 \pm 5.6$ & $16.7 \pm 8.2$ & $13.6 \pm 5.0$ & $20.7 \pm 9.1$ & $18.2 \pm 11.9$ & $15.9 \pm 7.9$ & $21.3 \pm 6.1$ \\
\hline
\end{tabular}

volume values as measured during the therapy. Changes in functional and static CT values between responders and nonresponders were tested by a $t$ test for unpaired samples. To detect any relationship between tumor volume and PCT parameters, Spearman correlation coefficients were calculated. ROC curves were calculated to assess the predictive value of the baseline and follow-up functional and morphologic parameters for the tumor response to chemoradiation. The mentioned thresholds were optimized between sensitivity and specificity. All of the tests were 2-sided, and the results were considered significant at a probability level below $5 \%$. The results were analyzed and graphs performed by using a commercially available statistical package (MedCalc 10.0.0.0 for Windows; MedCalc Software, Mariakerke, Belgium).

\section{Results}

Sixty PCT examinations followed by 60 routine CECT examinations were performed without serious motion artifacts that could hamper the diagnosis and quantification of the perfusion-associated parameters. No adverse effects were observed after contrast agent administrations and PCT acquisitions. All patients completed the chemoradiotherapy according to the protocol. Fourteen patients were classified as early responders and 6 patients as early nonresponders. The illustrative example of a responder and a nonresponder are shown in Fig 1.

\section{Descriptive and Comparison Analysis of Perfusion Parameters and Tumor Volume}

Compared with the baseline examination $(25.3 \pm 10.4 \mathrm{~mL})$, responders showed a significant reduction in the primary tumor volume after $40 \mathrm{~Gy}(14.2 \pm 5.4 \mathrm{~mL} ; P=.014)$ and after 70 Gy $(12.8 \pm 6.5 \mathrm{~mL} ; P=.007)$ of chemoradiation, whereas the difference between the tumor volume at 40 and 70 Gy did not reach the level of statistical significance.

After stratifying the patients into 2 groups according to their response to chemoradiotherapy (Table 2), at 40 Gy and at $70 \mathrm{~Gy}$, a weak significant trend of reduction in the BF values was recorded in responders after $40 \mathrm{~Gy}(P=.04)$, which was more pronounced after $70 \mathrm{~Gy}(P=.01)$. In contrast, in nonresponding patients the $\mathrm{BF}$ values showed a nonsignificant trend of elevation at $40 \mathrm{~Gy}$ (compared with the baseline values, $P=.06$ ) and did not change any further.

Whereas in responders the dynamics of changes in the BV values showed a reduction after $40 \mathrm{~Gy}$, which was still constant 


\begin{tabular}{|c|c|c|c|c|c|c|c|}
\hline Value & Criterion & AUC & $P$ & Sensitivity, $\%$ & Specificity, \% & Positive LR & Negative LR \\
\hline Baseline BF & $>70.4$ & 0.54 & .4 & 57.3 & 80.0 & 3.0 & 0.6 \\
\hline BF (40 Gy) & $\leq 74.4$ & 0.82 & .03 & 90.0 & 80.0 & 4.4 & 0.2 \\
\hline Baseline BV & $\geq 5.0$ & 0.83 & .01 & 59.5 & 100 & & 0.4 \\
\hline BV (40 Gy) & $\leq 6.9$ & 0.80 & .02 & 90 & 80 & 5.2 & 0.0 \\
\hline Baseline MTT & $\leq 9.0$ & 0.60 & .8 & 71.4 & 60 & 1.8 & 0.5 \\
\hline MTT (40 Gy) & $\leq 9.2$ & 0.83 & .04 & 100 & 60 & 2.4 & 0.0 \\
\hline Baseline PS & $>10.0$ & 0.63 & .1 & 100 & 60 & 2.2 & 0.0 \\
\hline PS (40 Gy) & $\leq 12.6$ & 0.80 & .06 & 65 & 100 & & 0.4 \\
\hline
\end{tabular}

Note:-The values of the provided criteria are the cutoff thresholds for predicting the defined early response. The statistically significant values ( $P \leq .05)$ are set in boldface italics; the values approaching statistical significance $(P=.06)$ are set in lightface italics. Values for $\mathrm{BF}$ are in $\mathrm{mL} / \mathrm{min} / 100 \mathrm{~g} ;$ for $\mathrm{BV}$, $\mathrm{mL} / 100 \mathrm{~mL}$; for $\mathrm{MTT}$, seconds; and for $\mathrm{PS}$, $\mathrm{mL} / \mathrm{min} / 100 \mathrm{~g}$.

after 70 Gy $(P=.04)$, in nonresponders there was a dosedependent nonsignificant elevation of the tumor BV observed.

The MTT in responders showed a slight temporary reduction at $40 \mathrm{~Gy}$, which was followed by a rebound as measured at $70 \mathrm{~Gy}$, nearly reaching the MTT values in the nonresponders. These changes in the MTT, however, were not statistically significant compared with the baseline values.

The PS values in the responders were almost stable after 40 Gy and nonsignificantly increased after 70 Gy $(P=.08)$, whereas in nonresponders they presented an elevation at 40 Gy, which was continued also after $70 \mathrm{~Gy}$ (0 Gy versus $70 \mathrm{~Gy}$, $P=.03)$. The descriptive statistics of the perfusion-associated parameters measurements as obtained at 3 time points are summarized in Table 2 .

\section{Correlation Analysis}

Regarding the interaction between morphologic and functional parameters in the pooled patient population (20 patients), a relationship existed also between baseline tumor volume and follow-up tumor volume at $40 \mathrm{~Gy}(r=0.82, P=$ $.001)$ and at $70 \mathrm{~Gy}(r=0.6, P=.03)$. Interrelationship was evident also between baseline tumor volume in the responders and the follow-up volume at $40 \mathrm{~Gy}(r=0.93, P=.001)$ as well as at $70 \mathrm{~Gy}(r=0.79, P=.03)$.

The tumor volume correlated only with the PS values at 40 Gy $(r=0.57, P=.04)$ as well as at $70 \mathrm{~Gy}(r=0.72, P=.006)$. In the responders, the tumor volume and PS values showed a significant negative correlation at $0 \mathrm{~Gy}(r=-0.74, P=.04)$, $40 \mathrm{~Gy}(r=-0.73, P=.05)$, and $70 \mathrm{~Gy}(r=-0.84, P=.03)$.

With regard to the BF values during the follow-up, statistically significant correlation was found between the BF value at $40 \mathrm{~Gy}$ and at $70 \mathrm{~Gy}(r=0.82, P=.0004)$, whereas baseline $\mathrm{BV}$ values correlated with the BV values at $40 \mathrm{~Gy}(r=0.51, P=$ $.06)$ and at $70 \mathrm{~Gy}(r=0.78, P=.004)$. However, no association was found between MTT values as measured at different time points during the therapy.

\section{ROC Curve Analysis}

The ROC curve analysis (Table 3) showed that among the baseline values of studied perfusion parameters, only BV can be used as a predictor of short-term tumor response with a sensitivity of $60 \%$ and specificity of $100 \%(P=.01)$. At the time point after completion of $40 \mathrm{~Gy}$ of concomitant chemoradiation, $\mathrm{BF}$ (sensitivity $=88.7 \%$, specificity $=80 \% ; P=$ .03 ), BV (sensitivity $=100 \%$, specificity $=90 \% ; P=.02$ ), and MTT (sensitivity $=90 \%$, specificity $=60 \% ; P=.04$ ) were shown to be helpful for prediction of response of the primary tumor to therapy.

\section{Discussion}

In our patients, although the tumor volume was reduced after chemoradiation, interestingly, the morphologic changes were not accompanied by variations in the functional parameters of any statistical significance, presumably due to the different trends in perfusion changes that were observed between individual patients. This also justified our choice to define the arbitrary criterion of early response and perform subanalysis to detect significant changes in the subgroups of early responders and nonresponders. Baseline BV proved to be a possible predictor for initial tumor response $(P=.01)$. This is in agreement with the results reported by Zima et al in patients receiving induction chemotherapy. ${ }^{9}$ Baseline $\mathrm{BF}$ was not found to hold a significant predictive role, opposed to the observations in patients undergoing radiation therapy ${ }^{6}$ or postoperative chemoradiation, ${ }^{12}$ and we think that the reason for these discrepancies might be the differences in treatment regimens and the observed end points (ie, tumor volume reduction, locoregional control, or survival probability).

In the responders, the dynamics of $\mathrm{BF}, \mathrm{BV}$, and PS values over the course of treatment showed either a pattern of significant constant reduction (BF), of significant initial reduction followed by a plateau (BV), or nonsignificant fluctuations (MTT and PS). These phenomena might be attributed to the cytotoxic effects of x-rays to the vascular endothelium cells, ${ }^{13}$ whereas concomitantly administered low-dose cisplatin chemotherapy per se has presumably no part in these processes. Chemotherapy is characterized by relatively little antivascular action in vivo, which is dose-dependent. ${ }^{14,15}$ When interpreting the fluctuations of the perfusion-associated parameters during the therapy course, hypoxia and intratumoral inflammation, especially after irradiation, play major roles. Such parameters have an impact on the perfusion-associated parameters, which cannot be predictable, as hypoxia may lead to perfusion decrease and inflammation may lead to high BV and PS values. Under these circumstances, PET/CT with specific tracers may serve as a surrogate in predicting short-term therapy outcome. Balancing of both chemotherapy and radiation therapy effects may have led to the observed pattern of changes in functional parameters in the responders. Because the increased permeability gives way to decreased vascular perfusion and fibrosis, ${ }^{16}$ the further reduction of BF after 70 Gy seems reasonable. Finally, the behavior of MTT values during the therapy may reflect the changes in physiology that resulted from the combined effect of chemotherapy and radiation therapy; that is, a short reduction in MTT at $40 \mathrm{~Gy}$, implying the vasodilation secondary to release of inflammatory cytokines caused by the radiation therapy, followed by subsequent pro- 
longation of MTT (at $70 \mathrm{~Gy}$ ) as a result of tumor endothelial cell apoptosis and fibrotic changes in the vessels and perivascular interstitial space. ${ }^{17}$

In the present study, the nonresponders demonstrated an acute increase of BF, BV, and PS, which resembles the changes that are described in lung cancer treated with radiation therapy. ${ }^{18}$ These changes were attributed to the up-regulation of VEGF in tumor and stromal cells and to the expression of endothelial nitric oxide that may have resulted in increased perfusion, opening up of previously nonperfused vessels, and in neoangiogenesis. ${ }^{19,20}$ However, VEGF promotes survival of endothelial cells and thereby radiation resistance. ${ }^{21}$ Ionizing irradiation may therefore contribute, through the induction of the VEGF and its receptors in residual tumor cells, to undesired effects. In the nonresponders, it seems that chemotherapy might not offset these undesired effects and, therefore, the patients did not experience local tumor shrinkage during concomitant chemoradiation. It is unknown whether late effects of chemoradiation (4-6 weeks after completion) in these patients may take place and contribute to local tumor control. The MTT values in the nonresponders showed a plateau during the therapy that may be traced back to the fact that the MTT is a "lumped" parameter and measures the transit time of the contrast agent not only through the vasculature but through the tissue voxel as well. Nonspecific changes in the tissue voxel may "contaminate" this parameter and lead to such results. Moreover, the applied postprocessing software is based on the central volume theorem (in which MTT $=\mathrm{BF}$ / $\mathrm{BV}$ ) and, thus, an increase in both BF and BV does not result in a change of MTT.

We sought to evaluate the predictive value of the perfusionassociated parameters for the initial response. In accordance with other authors investigating this issue in patients receiving induction chemotherapy ${ }^{8,9}$ whose elevated BF and BV values had a positive predictive role, our ROC analysis showed $100 \%$ specificity for tumor response in those patients with baseline $\mathrm{BV} \geq 5.0 \mathrm{~mL} / 100 \mathrm{~mL}$ tissue. Moreover, our results add to the recent observation that hyperemic tumors may respond better than tumors with low BV (and, thus, oxygenation) to concomitant chemoradiation as assessed by perfusion-weighted MR imaging ${ }^{10}$ or by PCT. ${ }^{12}$ Combined with new evidence suggesting a significant positive correlation between microvascular attenuation (which is supposed to be a local outcome predictor) and BV values, ${ }^{22,23}$ our results highlight the potential role of BV as a surrogate predictor for local outcome. Unlike the baseline measurements, the reestimation of PCT parameters at $40 \mathrm{~Gy}$ and at $70 \mathrm{~Gy}$ reveals that the decrease of vascularity (BF and BV values) and the decrease of the degree of neovascularization in the tumor (PS values) (attributable to the combined action of chemotherapy and radiation) are significant predictors for local tumor reduction. This emphasizes the value of the functional imaging at an early time in the organpreserving treatment protocols in locally and/or regionally advanced SCCHN. The specific pattern of changes in functional parameters may direct patients to an early salvage surgery or provoke readjustment of the irradiation or chemotherapy doses. However, the latter asks for significantly more reliable estimations of radiation sensitivity of primary tumor and nodal disease than those available at present. Tailoring radia- tion therapy dose to the degree of tumor sensibility would be extremely beneficial for the patients, sparing them from devastating treatment-related side effects. On the other hand, reduction of radiation dose or treatment intensity may lead to disease reappearance after seemingly successful treatment, when the predictive assay used was not accurate enough.

There are limitations in the present study that one should be aware of when discussing its results. First, the number of patients is low and does not allow firm conclusions. Furthermore, the estimation of the perfusion-associated parameters was restricted to $2.4 \mathrm{~cm}$, which is a known drawback of the PCT imaging compared with perfusion-weighted MR imaging, while the gross tumor volume calculation was based on more sections. However, by placing the PCT sections in the region of the largest tumor diameter we sought to minimize this pitfall. Additionally, the restrictions in area coverage did not allow us to estimate the gross nodal volume in all cases; this was the reason for its exclusion from analysis. The different perfusion characteristics between primary tumor and metastatic nodes, as being assessed recently with perfusionweighted MR imaging, ${ }^{10}$ do not allow an extrapolation of our results obtained from primary tumors to regional disease. Advanced stage is a union of both $\mathrm{T}$ stage and $\mathrm{N}$ stage, and thus locoregional control seems to be better assessed by perfusionweighted MR imaging or PET/CT. The perfusion parameters were calculated in ROIs that covered the whole tumor sections, which does not exclude microscopic necrotic foci and may falsely average the significant spatial heterogeneity of the tumor. Initial evidence on the estimation of maximum values inside the ROI might help to address this problem in future studies. ${ }^{12}$ Our hypothesis that PCT can detect changes in perfusion parameters during the course of the therapy implies a good repeatability of the PCT, which was not assessed in our patients, as this repeatability has previously proven to be satisfactory to excellent. ${ }^{24}$ The design of our study did not allow an estimation of locoregional tumor control after 1 or 2 years, which is certainly a more reliable criterion of therapy effectiveness and justifies the predictive value of the PCT. Nonetheless, our next step is to follow these patients and evaluate the predictive role of PCT in our future studies.

In conclusion, we demonstrated that PCT is clinically feasible for monitoring the effects of concomitant chemoradiotherapy in locoregionally advanced SCCHN. The results support the hypothesis that PCT can detect the dynamics in functional parameters during the course of the therapy and that some of them might also be used to predict early tumor response to applied therapy. Future studies may reinforce this evidence and lead to creation of a predictive model that could be of value in the clinical setting as a tool to tailor the therapy according to patient-specific tumor perfusion characteristics.

\section{References}

1. Haddad RI, Shin DM. Recent advances in head and neck cancer. N Engl J Med 2008;359:1143-54

2. Pignon JP, Bourhis J, Domenge C, et al. Chemotherapy added to locoregiona treatment for head and neck squamous-cell carcinoma: three meta-analyses of updated individual data. MACH-NC Collaborative Group. Meta-Analysis of Chemotherapy on Head and Neck Cancer. Lancet 2000;355:949-55

3. Bourhis J, Calais G, Lapeyre M, et al. Concomitant radiochemotherapy or 
accelerated radiotherapy: analysis of two randomized trials of the French Head and Neck Cancer Group (GORTEC). Semin Oncol 2004;31:822-26

4. Hermans R, Lambin P, Van den Bogaert W, et al. Non-invasive tumour perfusion measurement by dynamic CT: preliminary results. Radiother Oncol 1997;44:159-62

5. Bisdas S, Baghi M, Smolarz A, et al. Quantitative measurements of perfusion and permeability of oropharyngeal and oral cavity cancer, recurrent disease, and associated lymph nodes using first-pass contrast-enhanced computed tomography studies. Invest Radiol 2007;42:172-79

6. Hermans R, Meijerink M, Van den Bogaert W, et al. Tumor perfusion rate determined noninvasively by dynamic computed tomography predicts outcome in head-and-neck cancer after radiotherapy. Int J Radiat Oncol Biol Phys 2003;57:1351-56

7. Hoskin PJ, Saunders MI, Goodchild K, et al. Dynamic contrast enhanced magnetic resonance scanning as a predictor of response to accelerated radiotherapy for advanced head and neck cancer. Br J Radiol 1999;72:1093-98

8. Gandhi D, Chepeha DB, Miller T, et al. Correlation between initial and early follow-up CT perfusion parameters with endoscopic tumor response in patients with advanced squamous cell carcinomas of the oropharynx treated with organ-preservation therapy. AJNR Am J Neuroradiol 2006;27:101-06

9. Zima A, Carlos R, Gandhi D, et al. Can pretreatment CT perfusion predict response of advanced squamous cell carcinoma of the upper aerodigestive tract treated with induction chemotherapy? AJNR Am J Neuroradiol 2007;28:328-34

10. Cao Y, Popovtzer A, Li D, et al. Early prediction of outcome in advanced headand-neck cancer based on tumor blood volume alterations during therapy: a prospective study. Int J Radiat Oncol Biol Phys 2008;72:1287-90

11. Wiggenraad R, Mast M, van Santvoort J, et al. ConPas: a 3-D conformal parotid gland-sparing irradiation technique for bilateral neck treatment as an alternative to IMRT. Strahlenther Onkol 2005;181:673-82

12. Bisdas S, Nguyen SA, Anand SK, et al. Outcome prediction after surgery and chemoradiation of squamous cell carcinoma in the oral cavity, oropharynx, and hypopharynx: use of baseline perfusion CT microcirculatory parameters vs. tumor volume. Int J Radiat Oncol Biol Phys 2009;73:1313-18

13. Billis W, Fuks Z, Kolesnick R. Signaling in and regulation of ionizing radia- tion-induced apoptosis in endothelial cells. Recent Prog Horm Res 1998;53:8592, discussion 93

14. Cemazar M, Parkins CS, Holder AL, et al. Electroporation of human microvascular endothelial cells: evidence for an anti-vascular mechanism of electrochemotherapy. Br J Cancer 2001;84:565-70

15. Clements MK, Jones CB, Cumming M, et al. Antiangiogenic potential of camptothecin and topotecan. Cancer Chemother Pharmacol 1999;44:411-16

16. Cooper JS, Fu K, Marks J, et al. Late effects of radiation therapy in the head and neck region. Int J Radiat Oncol Biol Phys 1995;31:1141-64

17. Garcia-Barros M, Paris F, Cordon-Cardo C, et al. Tumor response to radiotherapy regulated by endothelial cell apoptosis. Science 2003;300:1155-59

18. Ng QS, Goh V, Milner J, et al. Acute tumor vascular effects following fractionated radiotherapy in human lung cancer: in vivo whole tumor assessment using volumetric perfusion computed tomography. Int J Radiat Oncol Biol Phys 2007;67:417-24

19. Ando S, Nojima K, Majima H, et al. Evidence for mRNA expression of vascular endothelial growth factor by $\mathrm{x}$-ray irradiation in a lung squamous carcinoma cell line. Cancer Lett 1998;132:75-80

20. Koutsimpelas D, Brieger J, Kim DW, et al. Proangiogenic effects of ionizing irradiation on squamous cell carcinoma of the hypopharynx. Auris Nasus Larynx 2008;35:369-75

21. Gupta VK, Jaskowiak NT, Beckett MA, et al. Vascular endothelial growth factor enhances endothelial cell survival and tumor radioresistance. Cancer J 2002;8:47-54

22. Ash L, Teknos TN, Gandhi D, et al. Head and neck squamous cell carcinoma: CT perfusion can help noninvasively predict intratumoral microvessel density. Radiology 2009;251:422-28

23. Kamijo T, Yokose T, Hasebe T, et al. Potential role of microvessel density in predicting radiosensitivity of $\mathrm{T} 1$ and $\mathrm{T} 2$ stage laryngeal squamous cell carcinoma treated with radiotherapy. Clin Cancer Res 2000;6:3159-65

24. Bisdas S, Surlan-Popovic K, Didanovic V, et al. Functional CT of squamous cel carcinoma in the head and neck: repeatability of tumor and muscle quantitative measurements, inter- and intra-observer agreement. Eur Radiol 2008;18: $2241-50$ 